\title{
INFLUÊNCIA DA TEMPERATURA NA PRODUÇÃO E BEM-ESTAR DE SUÍNOS
}

Nariane Coelho de Oliveira', Milena de Lima Vieira', Wallacy Barbacena Rosa dos Santos², Leonardo Batista Pedroso², Jeferson Corrêa Ribeiro², Andréia Santos Cezário², Eliandra Maria Bianchini Oliveira², Crislaine Messias de Souza²

${ }^{1}$ Instituto Federal de Educação, Ciência e Tecnologia Goiano - IFGOIANO, Curso de Zootecnia, Morrinhos, GO. ${ }^{2}$ Instituto Federal de Educação, Ciência e Tecnologia Goiano - IFGOIANO, Dept. de Zootecnia, Morrinhos, GO. E-mail: narianecoelho@gmail.com

\section{RESUMO}

A resposta dos suínos ao estresse térmico consiste, principalmente, na intensificação da dissipação de calor por evaporação pelo trato respiratório, seguida da redução no consumo de alimentos, com consequente redução na ingestão de energia, constituindo um esforço do animal em reduzir a produção de calor metabólico. Muitas dessas respostas fisiológicas e metabólicas ocasionam menores taxas de crescimento, baixa eficiência de utilização do alimento e alterações na composição de carcaça. Os suínos são animais com excelentes níveis de produção e reprodução, porém, um dos desafios da suinocultura está relacionado principalmente com a máxima exploração de seu potencial genético. Quando confinados em sistemas intensivos, os animais são postos a condições adversas, assim, mudam o seu comportamento de uma forma geral e principalmente em relação a ingestão de alimentos, que é alterada, ocorrendo então queda em seus índices zootécnicos, principalmente pelas alterações nos hábitos de alimentação. Desta forma, objetivou-se com esta revisão apresentar as possíveis influências da variação de temperatura sobre a produção e bem-estar dos suínos, e apontando as principais alterações fisiológicas que ocorrem com suínos submetidos a estas condições.

Palavras-chave: desempenho animal; estresse térmico; termoneutralidade.

\section{INFLUENCE OF TEMPERATURE IN SWINE PRODUCTION AND WELL-BEING}

\begin{abstract}
The response of pigs to thermal stress consists, mainly, of the intensification of heat dissipation by evaporation through the respiratory tract, followed by reduction in food consumption, with a consequent reduction in energy intake, constituting an effort by the animal to reduce heat production metabolic. Many of these physiological and metabolic responses lead to lower growth rates, poor feed utilization efficiency and changes in carcass composition. Pigs are animals with excellent levels of production and reproduction, but one of the challenges of swine farming is mainly related to maximum exploitation of their genetic potential. When confined in intensive systems, the animals are put under adverse conditions, thus, they change their behavior in a general way and mainly in relation to the food intake, which is changed, occurring then fall in their zootechnical indexes, mainly by the changes in the habits feed. Thus, the objective of this work is to present a review on the influence of temperature variation on the production and well-being of pigs, and pointing out the main physiological changes that occur with pigs submitted to these conditions.
\end{abstract}

Keywords: animal performance; thermal stress; thermoneutrality. 


\section{INTRODUÇÃO}

A suinocultura em regiões de produção intensiva de suínos, tem como objetivo gerar movimentação da economia e apresenta uma importância social e cultural. Porém, em regiões não tradicionais na atividade, uma produção baixa pode significar alternativa secundaria de renda para a população (FERREIRA, 2012a).

De acordo com Seganfredo et al. (2007), a atividade suinícola apresenta um rebanho mundial de 787 milhões de cabeças e representa 40\% do total da carne consumida, assim sendo a principal fonte de proteína animal consumida no mundo. A produtividade dos sistemas de criação nacionais tem aumentado, sobretudo com o advento de novas tecnologias e com os conhecimentos aprofundados sobre nutrição, fisiologia e sanidade dos suínos.

Todavia, por ser um país tropical, caracterizado por altas temperaturas, prejudiciais à criação de suínos, o ambiente térmico brasileiro é determinante sobre o sistema produtivo e, muitas vezes, constitui-se no responsável pelo desenvolvimento subótimo do plantel (MANNO et al., 2005).

Para Rodrigues et al. (2010), os suínos, são animais com excelentes níveis de produção e reprodução, porém, um dos desafios da suinocultura está relacionado principalmente com a máxima exploração de seu potencial genético, uma vez que, as principais linhagens e raças exploradas no Brasil são basicamente provenientes de raças europeias e norte asiáticas, adaptadas às condições mais frias de clima (ZANGERONIMO et al., 2015).

Assim, o estresse por calor representa um dos principais limitantes da produtividade no Brasil, onde regiões tropicais predominam em sua maior parte. O conhecimento da capacidade fisiológica de adaptação dos animais a estas condições é importante, para assegurar a máxima produtividade do plantel que por sua vez está ligada ao bem-estar dos animais (NUNES et al., 2016).

Os suínos são animais homeotérmicos, assim, esses animais podem apresentar melhor desempenho se estiverem em sua zona de conforto térmico, que compreende a faixa de temperatura ambiente efetiva, na qual o calor produzido durante os processos de mantença e de produção é igual ao calor perdido para o ambiente térmico, sem a necessidade de aumentar a taxa de produção de calor metabólico (MOUT, 1968).

Segundo Centurión (2012), o Brasil é um dos maiores produtores mundiais de carne suína, porém, apresenta inúmeras perdas produtivas devido ao fato de que a maioria das instalações não possui recursos de climatização adequados, geralmente obtendo limitada eficiência, seja por causa do custo de implementação e manutenção destes sistemas, ou do custo de energia elétrica. 0 clima do Brasil apresenta grande variação e tipologia, predispondo a ocorrência de verões com temperaturas e umidades elevadas, elementos meteorológicos que podem influir na produtividade agropecuária (VALE, 2008).

O estresse por calor é comum na grande maioria das criações de suínos no Brasil, já que a genética destes animais inclui raças adaptadas principalmente às condições temperadas de clima. Desta forma, objetivou-se com esta revisão apresentar as possíveis influências da variação de temperatura sobre a produção e bem-estar dos suínos, e apontando as principais alterações fisiológicas que ocorrem com suínos submetidos a estas condições.

\section{Bioclimatologia animal e seus aspectos}

Para Bridi (2010), alguns aspectos ambientais e fisiológicos, influenciam o conceito de bioclimatologia, deixando-o bastante amplo, pois, inclui uma somatória de elementos que afetam o desenvolvimento dos animais. Segundo Cordeiro et al. (2014), os componentes ambientais podem ser divididos em físicos (temperatura, umidade, ventilação, tipo das instalações), sociais (hierarquia, tamanho e composição do grupo, presença ou ausência de animais estranhos) e manejo (dietas, formas de arraçoamento, desmame). 
A redução na produtividade em sua maioria tem origem nos fatores ambientais externos e o microclima dentro das instalações, onde, ambos exercem efeitos diretos e indiretos sobre a produção animal em todas as fases de produção. O conhecimento das respostas ou adaptações fisiológicas, físicas e comportamentais dos animais relacionados ao ambiente térmico nos permite a tomada de medidas e/ou alteração de manejo, da nutrição, instalações e equipamentos, objetivando a maximização da atividade (BRIDI, 2006).

O suíno é considerado um agente modificador do meio em que vive, seja através da geração de calor, vapor d'água, fezes, urina ou como foco de desenvolvimento de patógenos. Nestas condições, o verdadeiro ambiente passa a ser aquele reinante no interior da edificação ou aquele cujo ar os animais respiram (PANDORFI, 2005).

\section{Principais raças suínas no Brasil}

De acordo com Cavalcanti (2000), os primeiros suínos do Brasil foram provenientes da Península Ibérica no período do descobrimento do Brasil. Dentro de uma mesma raça, existem animais bons e ruins, quando pensamos em desempenho de produtividade, o ideal é que o produtor escolha corretamente a raça, para que haja um manejo correto e adequado. Nenhuma raça nacional possui associação ou livro de registros, são animais de baixa produção, porém, rústicos e bem adaptados e, as raças naturalizadas mais representativas e reconhecidas no Brasil, são o Piau, Nilo, Canastra, Canastrão e Caruncho (FERREIRA, 2012b).

Entretanto, o melhoramento genético animal, fez-se necessário, a partir do século XX, quando a demanda da produção animal aumentou, tanto no Brasil como na Europa, assim no Brasil entre 1930 e 1960 começou a importação de raças exóticas com melhores desempenhos zootécnicos, como, Duroc, Pietrain, Landrace, Hampshire, Wessex, Large White, Large Black, Tamworth e Berkshire. Esse fato contribuiu bastante para a redução da variabilidade genética das linhagens de suínos presente no país (CAVALCANTI, 2000).

Grande parte dessas raças exóticas são provenientes da Europa ou América do Norte, ou seja, esses animais são adaptados a locais de clima frio, portanto, o estresse térmico é considerado um limitante da produtividade desses animais no Brasil, considerando que o clima é predominantemente tropical. Assim, fazendo-se necessário o entendimento da capacidade fisiológica de adaptação dos animais a condições adversas, dando subsídio para assegurar a máxima produtividade, que está intimamente ligada ao bem-estar dos animais (DOS SANTOS et al., 2014).

\section{Bem-estar na suinocultura}

Atualmente, o termo "bem-estar" está amplamente difundido, não só visando as melhores condições humanitárias de criação, mas também o aumento da produtividade do rebanho, ou seja, animais em adequadas condições de ambiente, são animais que possuem ambiente e instalações com ótimas condições de temperatura e umidade, além de um adequado convívio social (CARVALHO et al., 2013).

Dessa forma, é possível apresentar em sua maioria uma melhor saúde e melhores condições de desenvolvimento corporal, e consequentemente, menores gastos com medicamentos e melhor eficiência alimentar podem ser observados, afetando diretamente o custo de produção dos animais (RODRIGUES et al., 2010).

Para Braun (2000) na suinocultura, e em toda produção animal, cresce diariamente, até mesmo como exigência do mercado consumidor, a preocupação com o bem-estar animal, durante o seu já curto ciclo de vida imposto pela necessidade e desejo do consumo humano, principalmente em países de Primeiro Mundo, e também no Brasil, cada vez mais, a sociedade vem exigindo dos criadores, dos transportadores e da indústria, medidas que aliviem o "stress" e o sofrimento dos animais. 
O bem-estar animal teve uma maior importância na década de 1980, quando as análises não invasivas, como a utilização de imagens para avaliar o comportamento dos animais, se desenvolveu rapidamente, proporcionando melhores avaliações sobre a produtividade, e atualmente é crescente o número de pesquisas relacionadas ao bem-estar e comportamento animal, visando sempre melhorias para a condição de vida dos animais (FERREIRA; SABINO, 2013).

Falta de bem-estar durante a vida do animal também pode resultar em carne de qualidade inferior, assim, confinamento intensivo, estresse térmico, isolamento social, ausência de substrato, fome, alta densidade, agressão de animais dominantes, monotonia do ambiente, mutilação, baixa qualidade do ar, são todos fatores estressores que podem levar os animais a redirecionar o seu comportamento natural para "vícios", estereótipos ou comportamentos anômalos (MACHADO FILHO, 2000).

Para Baptista et al. (2011) o perfil do produtor brasileiro, mesmo que ainda discreto, vem sofrendo algumas mudanças, pois a busca da sociedade e do mercado externo por produtos éticos e de qualidade tem conquistado alguns progressos na produção de animais, o que torna necessário estabelecer critérios que avaliem o bem-estar dos suínos em seus sistemas de criação.

\section{Termorregulação e estresse térmico}

Os suínos são animais homeotérmicos, ou seja, tem a capacidade de manter a temperatura corporal dentro de certos limites, independente da variação da temperatura ambiente, assim, só apresentam máximo desempenho quando mantidos em ambiente térmico confortável, representado por uma faixa de temperatura em que os processos termorregulatórios são mínimos, com utilização total da energia líquida para deposição de tecidos (BERTON, 2013).

Segundo Bridi (2006), os suínos apresentam o aparelho termorregulador pouco desenvolvido. São animais sensíveis ao frio quando pequenos e sensíveis ao calor quando adultos, o que dificulta a sua adaptação aos trópicos. A dificuldade de se adaptar ao calor é devido principalmente ao seu elevado metabolismo, a capa de tecido adiposo subcutâneo e seu sistema termorregulador pouco desenvolvido e glândulas sudoríparas queratinizadas.

De acordo com Berton (2013), para a manutenção da temperatura corporal, os suínos utilizam formas de perda de calor, as quais são classificadas em sensível (não evaporativas) e latente (evaporativas), a transferência de calor sensível ocorre de três maneiras: condução, convecção e radiação.

Por não possuir glândulas sudoríparas funcionais a principal forma de liberação de calor pelos suínos é via latente, onde através da respiração ocorre perda de água por evaporação nas vias aéreas e também por evaporação de água em contato com a pele, proveniente dos dejetos líquidos ou existente sobre os pavimentos em que se deitam (ERHART et al., 2015; RICCl; DALLA COSTA, 2013).

O conforto térmico é dependente de diversos fatores, alguns ligados ao animal, como: peso, idade, estado fisiológico, tamanho do grupo, nível de alimentação e genética; e outros ligados ao ambiente como: temperatura, velocidade do vento, umidade relativa, tipo de piso e energia radiante (SARAIVA et al., 2003). De acordo com Bridi (2006), as faixas de conforto para leitões recém-nascidos varia entre 30 e $32^{\circ} \mathrm{C}$, enquanto que para porcas adultas em terminação essa faixa de conforto térmico diminui para 12 a $18^{\circ} \mathrm{C}$, e para ambos, a umidade relativa do ar deve-se encontrar entre 40 a $70 \%$ (ver tabela 1 ).

Estresse térmico é o desequilíbrio que ocorre no organismo do animal em resposta às condições ambientais adversas, tais como alta temperatura ambiente, alta umidade relativa do ar e alta radiação solar. 0 estresse por calor ocorre, então, quando a térmica que o animal recebe do ambiente, adicionada a carga calórica produzida pelo metabolismo, são maiores que a capacidade do animal em eliminar para o ambiente o estoque de calor excedente (RODRIGUES, 2014). 
Tabela 1. Temperaturas e umidades relativas ótimas e críticas para suínos na fase de crescimento e terminação.

Temperatura ótima

Suínos
Temperatura

crítica
Umidade relativa

\begin{tabular}{lcccccc} 
& Máx. & Mín. & Máx. & Mín. & Ótima (\%) & Crítica (\%) \\
\hline $20-35 \mathrm{~kg}$ & 20 & 18 & 27 & 8 & & \\
$35-60 \mathrm{~kg}$ & 18 & 16 & 27 & 5 & 70 & $<40>90$ \\
$60-100 \mathrm{~kg}$ & 18 & 12 & 27 & 4 & &
\end{tabular}

Adaptado: Berton, 2013.

Quando os suínos são expostos a temperaturas adversas, eles ficam estressados não só pela alteração da temperatura corporal, como também pela complexidade dos processos dissipadores e geradores de calor, que são processos metabólicos que requerem energia, nos quais a evaporação da umidade do aparelho respiratório é o mecanismo primário utilizado pelos animais para dissipar o excesso de calor corporal em um ambiente quente (CENTURIÓN, 2012). A utilização correta de processos e equipamentos que auxiliam o controle da temperatura, geralmente são eficazes na diminuição do estresse térmico, que por sua vez, é a maior causa de perdas produtivas em países tropicais.

\section{Frequência Cardíaca (FC)}

Agostini et al. (2011), com o objetivo de avaliar a inclusão de diferentes níveis de ractopamina em rações de suínos em fase de terminação, durante 21 dias pré-abate, sobre as características de desempenho, carcaça, qualidade de carne e sobre parâmetros fisiológicos e sanguíneos indicadores de bem-estar, concluíram que o aumento na frequência cardíaca nos animais tratados com ractopamina é consequência da elevação dos valores de adrenalina e noradrenalina séricos, e que a ractopamina, por ser similar às catecolaminas naturais, pode causar alterações fisiológicas nos animais, determinando aumento na frequência cardíaca, que por sua vez pode desencadear mudanças na frequência respiratória, alterando consequentemente o pH, as pressões parciais de $\mathrm{O} 2$ e $\mathrm{CO} 2$ e as concentrações dos íons $\mathrm{Na}+$ e $\mathrm{K}+$ no sangue, levando-os a quadros de estresse e à modificação do comportamento.

A frequência cardíaca se ajusta para atender à demanda de cada situação emocional (BAPTISTA et al., 2011). A frequência cardíaca dos suínos aumenta durante o transporte e principalmente nos manejos de embarque e desembarque (DALLA COSTA et al., 2006).

\section{Frequência Respiratória (FR)}

O primeiro indício de animais submetidos a estresse térmico é o aumento da frequência respiratória, que está relacionado com a intensidade e a duração do estresse que estão submetidos os animais. Esse mecanismo fisiológico promove a dissipação de calor por meio evaporativo, este aumento ocorre para estimular a perda evaporativa e manter o equilíbrio térmico corporal, ou seja, um verdadeiro auxílio para refrescar o corpo (RODRIGUES, 2014). Este aumento representa a principal e mais eficiente forma de dissipar calor em suínos e aves submetidas a altas temperaturas (OLIVEIRA NETO et al., 2001). A frequência respiratória normal em suínos adultos varia entre 15 a 25 movimentos por minuto (YAN; YAMAMOTO, 2000). 
Tavares et al. (2000), em estudos utilizando machos castrados $(n=100)$, e mantidos em temperaturas de $22^{\circ} \mathrm{C}$ e $32^{\circ} \mathrm{C}$, os autores verificaram que houve aumento da frequência cardíaca nos animais mantidos à temperatura de $32^{\circ} \mathrm{C}$. Em estudo similar, foi observado um aumento na frequência respiratória, em temperatura ambiental de $32^{\circ} \mathrm{C}$ (machos castrados, Landrace $\mathrm{x}$ Large White, peso $29,94 \pm 0,49 \mathrm{~kg}, \mathrm{n}=36$ ), os autores concluíram que a frequência respiratória pode ser aumentada pelo estresse térmico em função da respiração ser um eficiente mecanismo fisiológico que atua na manutenção da termorregulação (MANNO et al., 2006).

\section{Temperatura retal}

Quando a temperatura ambiente se eleva acima da capacidade de reajuste fisiológico, o calor corporal retido é capaz de alterar o estado de homeotermia, sendo comum um incremento da temperatura retal, que se torna mais intenso com o grau de desvio da temperatura de conforto térmico (RODRIGUES, 2014).

A aferição da temperatura retal é utilizada, normalmente, como índice de adaptação fisiológica ao ambiente quente. Um aumento em seu valor significa que o animal está estocando calor, assim, o estresse térmico manifesta-se, pois seu aumento indica que os mecanismos de liberação de calor tornaram-se insuficientes para manter a homeotermia. A temperatura retal, representa a temperatura local e, embora nem sempre represente uma média da temperatura corporal profunda, é considerada a melhor para medir a temperatura corporal. Além disso, a temperatura retal atinge o equilíbrio mais lentamente do que em muitos outros locais, sendo uma boa indicadora de um verdadeiro estado estacionário (SWENSON; REECE, 1996).

\section{Mecanismos de adaptação ao calor (adaptações fisiológicas)}

Na suinocultura é possível observar que quando os animais são submetidos à situações de alta temperatura, eles utilizam mecanismos metabólicos que agem na manutenção da homeotermia, ou seja, a temperatura ambiental e o microclima das instalações influenciam na temperatura corporal e superficial do suíno (MANNO et al., 2006).

De acordo com Rodrigues (2014), as respostas fisiológicas adaptativas ao calor incluem vasodilatação periférica, aumento da taxa de produção de suor (taxa de sudorese), aumento da frequência respiratória, aumento da temperatura da pele e dos batimentos cardíacos, redução no metabolismo basal e energético e consequentemente redução no consumo de alimento (ver tabela 2).

Tabela 2. Efeito da temperatura ambiental acima de $22^{\circ} \mathrm{C}$ por $48 \mathrm{~h}$ em leitoas de $89 \mathrm{Kg}$.

\begin{tabular}{lcccc}
\hline Temperatura Ambiente & $22,7^{\circ} \mathrm{C}$ & $25,9{ }^{\circ} \mathrm{C}$ & $28,5^{\circ} \mathrm{C}$ & $31,4{ }^{\circ} \mathrm{C}$ \\
\hline Temperatura de Pele & 33,9 & 35,1 & 37,0 & 37.9 \\
Temperatura do Corpo & 39,0 & 39,1 & 39.5 & 40.4 \\
Frequência Respiratória/min & 27 & 51 & 85 & 112 \\
Taxa Cardíaca litro/min & 9,3 & 9,5 & 8,4 & 7,5 \\
Consumo g/dia & 2846 & 2340 & 1888 & 900 \\
\hline
\end{tabular}

Adaptado: Gilles et al., 1990.

Para Rodrigues (2014), além das adaptações fisiológicas citadas anteriormente, outro componente que também é utilizado para a indicação do estado de estresse dos animais é o peso dos órgãos, pois, a redução do peso dos órgãos causada pela temperatura elevada, provavelmente, é sinal de um ajuste fisiológico dos animais na tentativa de reduzir a produção de calor interno. 
De acordo com Mendes (2005), outro mecanismo fisiológico de controle da temperatura corporal é o fluxo sanguíneo. Em situações de elevada temperatura, este fluxo ocorre com maior intensidade do interior do núcleo corporal para a periferia, sendo responsável pela dissipação de calor por convecção e ocasionando alterações na temperatura da pele, assim, a medida da temperatura da pele é adotada como um indicador de estresse térmico.

Os suínos, submetidos a altas temperaturas, apresentam mudanças de comportamento, como, por exemplo, deitam-se de lado com o focinho em direção ao vento, para aumentar a taxa de troca térmica por convecção através da respiração. Outra mudança de comportamento é que, normalmente defecam em local mais isolado, porém, ocorrendo o calor excessivo, eles deitam sobre seus excrementos para fugir dessas condições, além da busca por água e locais úmidos (MÜLLER, 1982).

\section{Conforto térmico}

O conforto térmico ambiental era considerado um problema secundário, tanto do ponto de vista etológico quanto do produtivo, pois, acreditava-se que o desconforto térmico seria resolvido com o uso de condicionamento artificial, sem se considerar os custos e os problemas relacionados à implantação de um sistema climatizado (MANNO et al., 2005).

O ambiente térmico em uma instalação geralmente engloba os efeitos da radiação solar, da temperatura do ar, da umidade relativa do ar e da velocidade do vento. Dentre os elementos climáticos, a temperatura elevada associada à alta umidade relativa causa queda no desempenho do suíno, e interfere em seu bem-estar (PERDOMO, 1998).

A zona termoneutra, é a faixa de temperatura onde ocorre o mínimo de desperdício, e, é limitada pela temperatura crítica inferior, região onde o animal necessita aumentar a taxa de produção de calor para manter a homeotermia, e pela temperatura crítica superior, onde o animal deve perder calor para manter a temperatura corporal constante (SOUZA, 2002).

De acordo com Rodrigues (2014), cada fase de criação dos suínos, possui uma faixa de temperatura de conforto, onde não há nenhuma atividade metabólica para aquecer ou esfriar o animal, como exemplo é o caso de suínos de 20 a $60 \mathrm{Kg}$, a faixa de temperatura de $15{ }^{\circ} \mathrm{C}$ a 22 o $\mathrm{C}$ constitui a zona de termoneutralidade (Tabela 3), proporcionando o máximo de desempenho do animal.

Valores de temperatura ambiente muito diferente dos valores próximos à região de conforto térmico perturbam o mecanismo termodinâmico que os homeotermos têm de se proteger de extremos de temperatura e umidade relativa, levando ao desperdício de energia

e, consequentemente, refletindo nos números que medem o desempenho, pois dentro da zona termoneutra a energia fornecida pelos alimentos é utilizada no crescimento, manutenção e atividade física, fora da zona termoneutra, essa energia pode ser desviada para processos que visem manter a homeotermia (COLLIN et al., 2001).

Tais condições devem sempre ser levadas em consideração nas diferentes fases de criação dos suínos, procurando manter o máximo possível de bem-estar aos animais, associando sempre à máxima produtividade do plantel (RODRIGUES, 2014). 
Tabela 1. Zona de termoneutralidade dos suínos nas diversas fases de produção.

\begin{tabular}{|c|c|c|c|c|c|}
\hline \multirow{2}{*}{ Categoria } & \multicolumn{2}{|c|}{ Temperatura ideal $\left({ }^{\circ} \mathrm{C}\right)$} & \multicolumn{2}{|c|}{ Temperatura crítica $\left({ }^{\circ} \mathrm{C}\right)$} & \multirow{2}{*}{$\begin{array}{c}\text { Umidade relativa (\%) } \\
\text { Ótima }\end{array}$} \\
\hline & Máxima & Mínima & Máxima & Mínima & \\
\hline Matrizes & 18 & 12 & 30 & 0 & $50-70$ \\
\hline Leitões & 32 & 30 & 35 & 15 & 70 \\
\hline 1 semana & 28 & 27 & 35 & 15 & 70 \\
\hline 2 semanas & 26 & 25 & 35 & 13 & 70 \\
\hline 3 semanas & 24 & 22 & 35 & 13 & 70 \\
\hline 4 semanas & 22 & 21 & 31 & 10 & 70 \\
\hline \multicolumn{6}{|l|}{ Nascimento } \\
\hline 5 a 8 semanas & 22 & 20 & 30 & 08 & $50-70$ \\
\hline 20 a 30 kg & 20 & 28 & 27 & 08 & $50-70$ \\
\hline 30 a $60 \mathrm{~kg}$ & 18 & 16 & 27 & 05 & $50-70$ \\
\hline 60 a $100 \mathrm{~kg}$ & 18 & 12 & 27 & 05 & $50-70$ \\
\hline
\end{tabular}

Adaptado: Silva (2000).

Em relação ao conforto térmico dos suínos durante o transporte, Murray (2000) afirma que uma ventilação adequada durante o transporte é muito importante, e que alta temperatura e alta umidade no veículo de transporte aumentam o estresse e estão muitas vezes associadas ao aumento da mortalidade durante o transporte e carne PSE (pale, soft and exsudative), deve ser evitado o transporte na parte mais quente de um dia de verão, e em casos de temperaturas mais quentes, a densidade geralmente é ajustada para permitir mais espaço por animal.

\section{CONSIDERAÇÕES FINAIS}

É importante o conhecimento dos inúmeros fatores externos e internos (microclima) presentes na criação de suínos que podem exercer efeitos diretos e/ou indiretos sobre os animais em todas as fases de produção e acarretar redução na produtividade, com consequentes prejuízos econômicos no setor da suinocultura. Entender por que e como o ambiente influencia os suínos nos diferentes estádios de desenvolvimento e como estes animais respondem ao ambiente térmico e as variações climáticas, é de suma importância. No entanto, o efeito de um ambiente climático adequado ao animal, por si só, talvez não reflita de imediato numa melhora significativa na produção, pois há fatores como a genética, a nutrição e a sanidade do rebanho a serem considerados.

\section{REFERÊNCIAS}

AGOSTINI, P.S. et al. Efeito da ractopamina na performance e na fisiologia do suíno. Archivos de zootecnia, v. 60, n. 231, p. 659-670, 2011.

BAPTISTA, R.I.A.A.; BERTANI, G.R.; BARBOSA, C.N. Indicadores do bem-estar em suínos. Ciência Rural, v. 41, n. 10, p. 1823-1830, 2011. https://doi.org/10.1590/S0103-84782011005000133

BERTON, M. P. Ambiente controlado e não controlado no desempenho, comportamento e características de carcaça de suínos. 2013.

BRAUN, J.A. O bem-estar animal na suinocultura. In: 1 Conferência Internacional Virtual sobre Qualidade de Carne Suína. 2000. p. 1. 
BRIDI, A. M. Adaptação e aclimatação animal. Apostila de Bioclimatologia. Disponível em:< http://pt. slideshare.net/RmuloAlexandrinoSilva/apostila-conforto-termico2012> v. 5, p. 05-15, 2006. Acesso em: 20 de outubro de 2016.

BRIDI, A.M. Adaptação e aclimatação animal. UEL, Londrina, 2010.

BROOM, D.M. Indicators of poor welfare. British Veterinary Journal, London, v.142, p.524-526, 1986. https://doi.org/10.1016/0007-1935(86)90109-0

CARVALHO, C.M.C.; ANTUNES, R.C.; CARVALHO, A.P.; CAIRES, R.M. Bem estar na suinocultura. Revista Eletrônica Nutritime, v. 11, p. 2272-2286, 2013.

CAVALCANTI, S. S. Suinocultura dinâmica. Rome: FEP - MVZ Ed. Contagem, 2000. 494p.

CENTURIÓN, R. A. O. AMBIENTE TÉRMICO E BEM-ESTAR DE SUÍNOS NO PERÍODO DE DESCANSO PRÉ-ABATE. 2012. Tese de Doutorado. UNIVERSIDADE FEDERAL DA GRANDE DOURADOS.

COLLIN, A. et al. Effect of high temperature on feeding behaviour and heat production in grouphoused young pigs. British Journal of Nutrition, v. 86, n. 01, p. 63-70, 2001. https://doi.org/10.1079/BJN2001356

CORDEIRO, M.D.; MENEZES, T.Q.; PAULA, M.O. Capítulo 32- "Ambiência e Bem-Estar Animal na Produção de Aves e Suínos". Bruno Borges Deminicis \& Carla Braga Martins, p. 332, 2014.

DALLA COSTA, O.A. et al. Efeito do tempo de jejum dos suínos na granja sobre o bem-estar, medido pelo cortisol na saliva e pela frequência cardíaca, durante o manejo pré-abate. Concórdia: Embrapa Suínos e Aves, 2006. 3p. (Comunicado técnico, 439).

DOS SANTOS, C.F.M.; CONCEIÇÃO, G.C.; BRACHT, F. Porcos da metrópole e atuns da colônia: adaptação alimentar dos colonizadores europeus na América portuguesa quinhentista. Estudos Ibero-Americanos, v. 39, n. 2, p. 344-364, 2014 . https://doi.org/10.15448/1980864X.2013.2.13744

ERHART, A.I. et al. Prolificidade, peso ao nascer e número de tetos em raças de suínos de linhas maternas e paternas. 2015.

FERREIRA, R. A.; SABINO, L.A. Bem estar na suinocultura. Caderno de Ciências Agrárias. Montes Claros - MG, v. 5, n. 1, p. 115-122, 2013.

FERREIRA, R. A. Suinocultura manual prático de criação. Aprenda Fácil, Viçosa, p. 24 2012a.

FERREIRA, R. A. Suinocultura manual prático de criação. Aprenda Fácil, Viçosa, p. 101-105. 2012b.

MACHADO FILHO, L.C.P. Bem-estar de suínos e qualidade da carne: Uma visão Brasileira. In: 1 Conferência Internacional Virtual sobre Qualidade de Carne Suína. p. 34-40, 2000.

MANNO, M.C.; OLIVEIRA, R.F.M.; DONZELE, J.L. et al. Efeito da temperatura ambiente sobre o desempenho de suínos dos 15 aos 30kg. Revista Brasileira de Zootecnia, v.34, n.6, p.1963-1970, 2005. https://doi.org/10.1590/S1516-35982005000600021 
MANNO, M.C. et al. Efeitos da temperatura ambiente sobre o desempenho de suínos dos $\mathbf{3 0}$ aos 60kg. Revista Brasileira de Zootecnia, Viçosa, MG, v.35, n.2, p.471-477, 2006.

MENDES, A. S. Efeito do manejo da ventilação no ambiente de salas de maternidade para suínos. 2005. 89p. Dissertação (Mestrado em Agronomia) - Escola Superior de Agricultura "Luís de Queiroz", Piracicaba.

MOUNT, L.E. The climatic physiology of the pig. London: Edward Arnold. 1968. p.271.

MURRAY, A.C. Reduzindo perdas da porteira da granja até o abatedouro - Uma perspectiva Canadense. In: 1 Conferência Internacional Virtual sobre Qualidade de Carne Suína. p. 76-88, 2000.

MÜLLER, P.B. Bioclimatologia aplicada aos animais domésticos. 2ed. Posto Alegre: Sulina, 1982. $183 p$.

NUNES, M.L.A. et al. AVALIAÇÃO FISIOLÓGICA DE ESTRESSE POR CALOR EM PORCAS GESTANTES SUBMETIDAS A DIFERENTES SISTEMAS DE ALOJAMENTO EM CAMA E EM PISO DE CONCRETO. Engenharia Agrícola, v. 34, n. 1, 2016.

OLIVEIRA NETO, A. R. et al. Exigência de metionina + cistina para frangos de corte mantidos em ambiente de estresse de calor. In: REUNIÃO ANUAL DA SOCIEDADE BRASILEIRA DE ZOOTECNIA, 38., 2001, Piracicaba. Anais... Piracicaba: SBZ, 2001.

PANDORFI, H. Comportamento bioclimático de matrizes suínas em gestação e o uso de sistemas inteligentes na caracterização do ambiente produtivo: suinocultura de precisão. 2005. Tese de Doutorado. Universidade de São Paulo. https://doi.org/10.11606/T.11.2005.tde-09092005-145206

PERDOMO, C. C. Mecanismos de aclimatização de frangos de corte como forma de reduzir a mortalidade no inverno e verão. In: SIMPÓSIO INTERNACIONAL SOBRE INSTALAÇÕES E AMBIÊNCIA, 1998, Campinas. Anais... Campinas. 1998. p. 229-238.

RICCI, G.D.; DALLA COSTA, O. A. Abate humanitário de suínos. Revista de Ciências Agroveterinárias, v. 14, n. 3, p. 267-272, 2013. https://doi.org/10.5965/223811711432015267

RODRIGUES, N. E. B.; ZANGERONIMO, M. G.; FIALHO, E. T. Adaptações fisiológicas de suínos sob estresse térmico. Revista Eletrônica Nutritime, v. 7, p. 1197-1211, 2010.

RODRIGUES, N. E. B. Uso de rações modificadas para suínos em terminação mantidos em ambiente de conforto ou de calor. 2014.

SARAIVA, E.P.; OLIVEIRA, R.F.M.; DONZELE, J.L. et al. Níveis de Proteína Bruta em Rações para Suínos Machos Castrados em Fase Inicial de Crescimento, mantidos em Ambiente de baixa temperatura. Revista Brasileira de Zootecnia, v.32, n.6, p.1690-1696, 2003. https://doi.org/10.1590/S1516-35982003000700019

SEGANFREDO, M.A. et al. 1ed. Gestão ambiental na suinocultura. Embrapa Informação Tecnológica, 2007. p.17. 
SOUZA, P. Avaliação do índice de conforto térmico para matrizes suínas em gestação segundo as características do ambiente interno. Campinas: FEAGRI/UNICAMP, 2002. 103p. (Tese de Doutorado).

SWENSON, M.J.; REECE, W.O. Fisiologia dos animais domésticos. 11.ed. Rio de Janeiro: Guanabara Koogan, 1996. 856p.

TAVARES, S.L.S.; DONZELE, J.L.; OLIVEIRA, R.F.M.; FERREIRA, A.S. Influência da temperatura ambiente sobre o desempenho e os parâmetros fisiológicos de suínos machos castrados dos $\mathbf{3 0}$ aos $60 \mathrm{~kg}$. Revista Brasileira de Zootecnia, Viçosa, MG, v.29, n.1, p.199205, 2000.

VALE, M. M. Caracterização e previsão de ondas de calor com impacto na mortalidade de frangos de corte. Tese (Doutorado em Engenharia Agrícola). 101f, 2008, FEAGRI, Faculdade de Engenharia Agrícola, UNICAMP, Campinas, SP: [s.n.], 2008.

YAN, P.S; YAMAMOTO, S. Relationship between thermoregulatory responses and heat loss in piglets. Animal-Science -Journal. 2000, v. 71, n. 10, p. 505-509.

ZANGERONIMO, M.; OBERLENDER, G.; MURGAS, L. Efeito da nutrição na reprodução em marrãsRevisão de literatura. Revista Científica Eletrônica de Medicina Veterinária, v. 20, n. 1, p. 1-20, 2015. 